\title{
A protocol combining current- and voltage-clamp provides a novel and useful three-dimensional representation of cardiac action potential
}

\author{
Massimiliano Zaniboni, ${ }^{1,2}$ Francesca Cacciani ${ }^{1}$ \\ ${ }^{1}$ Department of Biosciences, University of Parma; ${ }^{2}$ Center of Excellence for Toxicological Research, \\ University of Parma, Italy
}

\begin{abstract}
A compact three-dimensional representation of cardiac action potential (AP) properties in terms of current source is presented here. The experimental protocol used to obtain such representation is based on the measure of instantaneous current-voltage relationships during the course of the AP. The procedure, which combines current- and voltage-clamps on patch clamped cardiac myocytes, has been previously applied to real cells, and then extended to computer simulations with cellular ventricular AP models. The three-dimensional AP representation allows to easily estimate membrane resistance during repolarization, a key factor for the modulation of ventricular repolarization. It also shows that, during late ventricular repolarization, membrane conductance becomes negative, i.e. repolarization is auto-regenerative. The novel AP representation is therefore a useful tool for both in vivo and in silico cardiac cellular electrophysiological investigations.
\end{abstract}

\section{Introduction}

The cardiac cellular action potential (AP) describes the electrical activity of heart cells by means of a function (membrane potential $V_{m}$ ) of a single variable (time). Though invaluable for the study of cardiac electrophysiology, this type of representation is intrinsically limited by the fact that substantially different membranes can display very similar or even identical APs, ${ }^{1,2}$ endowed with quite different dynamical properties. A compact representation of cardiac AP properties in terms of current source would be beneficial for cardiac electrophysiological research.

Correspondence: Massimiliano Zaniboni, Department of Biosciences, University of Parma, Parco Area delle Scienze 11A, 43124 Parma, Italy. Tel. +39.0521.902111 - Fax: +39.0521.347038.

E-mail: massimiliano.zaniboni@unipr.it

Key words: cardiac repolarization, cardiac action potential, membrane resistance, cardiac computational modelling.

(C) Copyright M. Zaniboni and F. Cacciani, 2014

Licensee PAGEPress, Italy

Journal of Biological Research 2014; 87:2134

doi:10.4081/jbr.2014.2134

This article is distributed under the terms of the Creative Commons Attribution Noncommercial License (by-nc 3.0) which permits any noncommercial use, distribution, and reproduction in any medium, provided the original author(s) and source are credited.
Based on previous patch clamp ${ }^{3}$ and modelling ${ }^{1,4-6}$ studies, we present here a protocol which combines current- and voltage-clamp in order to measure instantaneous current-voltage relationships during AP repolarization, and to generate three-dimensional time-voltagecurrent surfaces, which compactly describe several key dynamic features of the corresponding membrane. The in silico version of the protocol allows higher time and voltage (and therefore current) - resolution, and is applied here on a human ventricular AP model. ${ }^{7}$ The threedimensional AP representation allows to easily estimate membrane resistance (the inverse of the slope of $\mathrm{I}_{\mathrm{m}}-\mathrm{V}_{\mathrm{m}}$ slices) during repolarization, which is a key factor involved in its electrotonic modulation. The 3D surface also shows that, during late repolarization phase, membrane conductance becomes negative, which leads to the novel finding that, at this time, repolarization is auto-regenerative. A number of additional and relevant features concerning repolarization dynamics are compactly contained in the three-dimensional AP representation.

\section{Materials and Methods}

A preliminary and incomplete version of the procedure has been previously applied on real enzymatically isolated guinea pig cardiac ventricular myocytes (Figure 1). During the repolarization of an electrically elicited (current clamp) AP, and at different time delays after AP overshoot (see arrows in Figure 1A), the membrane was voltage clamped for $50 \mathrm{~ms}$ at the $\mathrm{V}_{\mathrm{m}}$ occupied by AP trajectory at that time $\left(\mathrm{V}_{\mathrm{mt}}\right)$, and, in subsequent runs, at $V_{\mathrm{mt}}-10 \mathrm{mV}$, and at $\mathrm{V}_{\mathrm{mt}}+10 \mathrm{mV}$. The ion current $\left(\mathrm{I}_{\text {ion }}\right)$ flowing during each step was recorded (Figure 1B) and allowed to draw instantaneous current-voltage relations at each repolarization time. The slope of each of these functions, as measured at $V_{\mathrm{mt}}$, provides the inverse of membrane input resistance $\left(\mathrm{R}_{\mathrm{m}}\right)$ at the corresponding time. The experimental protocol is here simulated on the Priebe \& Beuckelmann mathematical model ${ }^{7}$ of human cardiac ventricular AP, recompiled in its Matlab version by means of COR facility (http://cor.physiol.ox.ac.uk). The in silico version of the experimental procedure allows a much higher resolution in time, voltage, and therefore in ion current.

\section{Results}

In our simulations, voltage clamp steps were applied every $1 \mathrm{~ms}$ during AP repolarization (only 5 representative examples are reported in Figure $2 \mathrm{~A}$ ), and $\mathrm{V}_{\mathrm{m}}$ clamped, at each time, every $1 \mathrm{mV}$ starting from - 120 $\mathrm{mV}$ up to $+40 \mathrm{mV}$. From the $\mathrm{I}_{\mathrm{ion}}$ density generated by the Priebe \& Beuckelmann models equation system during each step (Figure 2B), the $\mathrm{I}_{\text {ion }}$ value (measured $1 \mathrm{~ms}$ after the onset of the step) was taken to construct an instantaneous $I_{\text {ion }}-V_{m}$ curve at any given time of AP repolariza- 

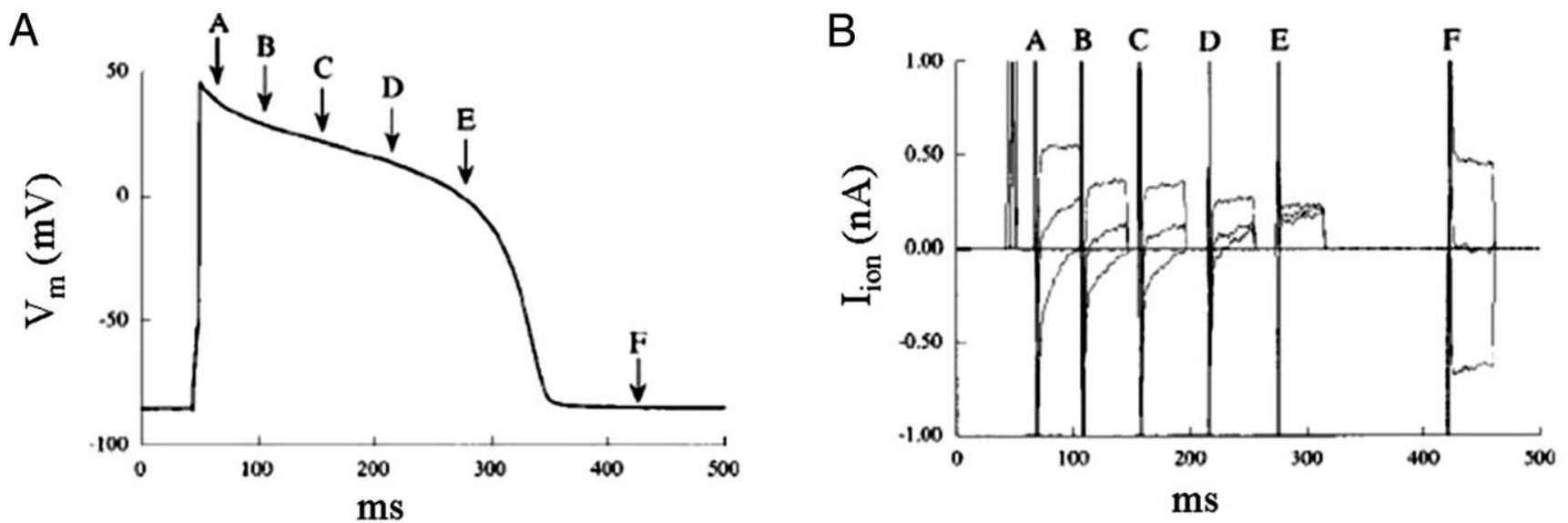

Figure 1. Combined current/voltage clamp protocol applied to a guinea pig ventricular cell. A) Arrows indicate time of switching to voltage clamp during an electrically elicited action potential; B) ion currents recorded during each voltage clamp (from Zaniboni $e t$ al. ${ }^{3}$ ).
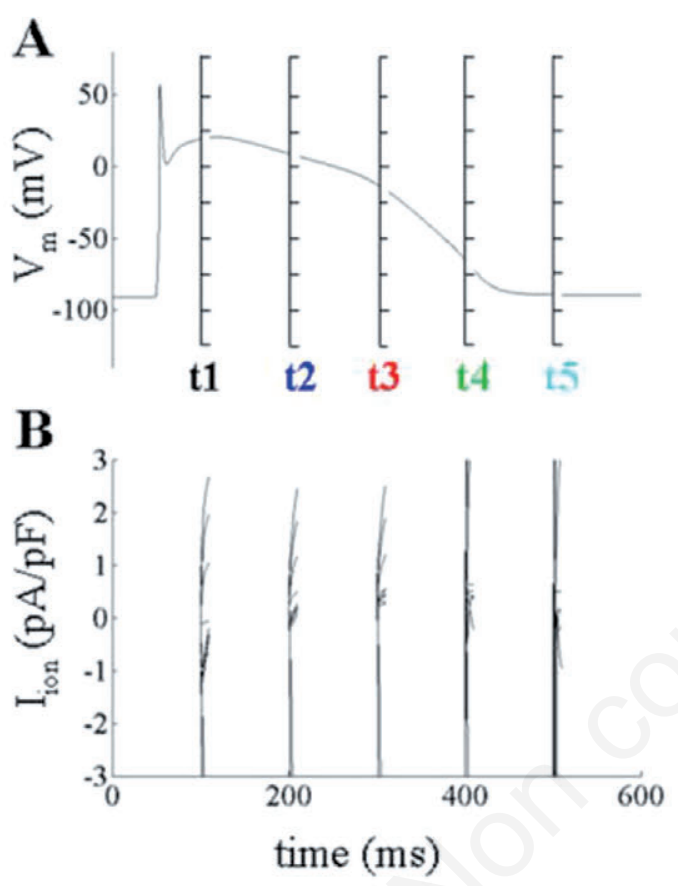

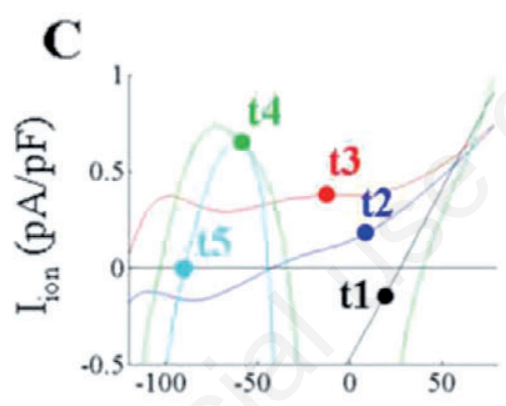

D

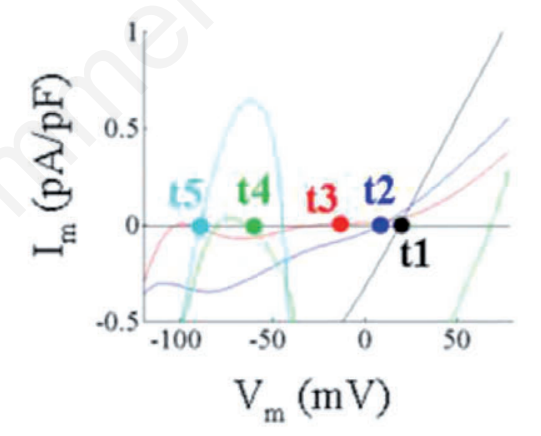

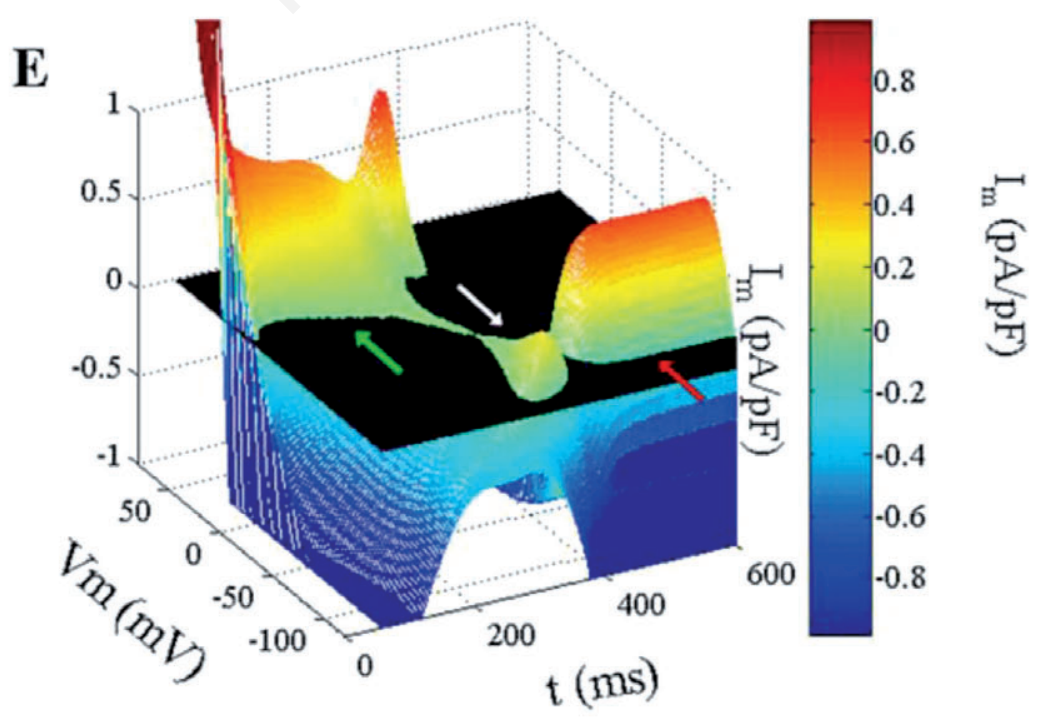

Figure 2. A) and B) represent the same protocol described in Figure 1, simulated here on the Priebe $\&$ Beuckelmann model. C) Representative examples of $I_{i o n}-V_{m}$ curves obtained from panels $A$ and $B ; D)$ representative examples of $I_{m}-V_{m}$ curves obtained by subtracting capacitive current from $I_{i o n}-V_{m}$ curves in (C). E) Reconstruction of the $I_{m}$ $V_{m}$-t surface from all the $I_{m}-V_{m}$ curves measured every $1 \mathrm{~ms}$. t1-t5=voltage clamp steps. 
tion (Figure 2C). Furthermore, the capacitive current $\left(\mathrm{I}_{\mathrm{C}}\right)$ density ($\mathrm{dV}_{\mathrm{m}} / \mathrm{dt}$ ), flowing at each time during $\mathrm{AP}$ repolarization, was added to $\mathrm{I}_{\text {ion }}$ for each $I_{\text {ion }}-V_{m}$ curve, leading to a collection of $I_{m}-V_{m}$ curves, where $I_{m}$ is membrane current $\left(\mathrm{I}_{\mathrm{m}}=\mathrm{I}_{\mathrm{C}}+\mathrm{I}_{\text {ion }}\right)$ density. The collection of $\mathrm{I}_{\mathrm{m}}-V_{\mathrm{m}}$ plots (see representative examples in Figure 2D) generates a 3D surface (Figure $2 \mathrm{E}$ ) where $\mathrm{x}$ is time, $\mathrm{y}$ is $\mathrm{V}_{\mathrm{m}}$ and $\mathrm{z}$ is $\mathrm{I}_{\mathrm{m}}$. The AP profile lies on the region where such surface intersects the $\mathrm{I}_{\mathrm{m}}=0$ plane. A useful way to represent the $I_{m}$ surface is through contour plots like that reported in Figure 3B, where, for the sake of simplicity, only the sign, positive (green colour) and negative (white colour), of $\mathrm{I}_{\mathrm{m}}$ values is reported. As mentioned above, the slope of $I_{m}-V_{m}$ slices, taken every $m s$, allows to easily derive the time course of membrane input resistance $\left(\mathrm{R}_{\mathrm{m}}\right)$ during AP repolarization (Figure 3C). Also, this type of representation clearly shows the time window when the slope of $I_{m}-V_{m}$ slices changes sign and becomes negative (delimited by vertical dotted blue lines in Figure 3 ). Since a negative conductance leads to a positive feedback between $V_{m}$ and $I_{m}$ changes, we call this time window auto-regenerative repolarization phase (ARRP).

\section{Discussion}

Despite the huge amount of studies performed over the years both at the cellular and tissue level, the electrophysiology of cardiac ventricular repolarization and its modulation is far to be fully elucidated. In recent years, the increasing availability of mathematical models of ventricular AP for different species makes it easier to test new hypotheses on this field, and also to elucidate key AP properties which are difficult to access in vivo. Along this line, we extended a complex combined current/voltage clamp protocol to a recently developed human ventricular AP model, in order to give a compact representation of membrane AP repolarization, which includes a novel finding that needs to be verified in vivo. Beside the possibility to easily measure membrane input resistance during repolarization, which is recognized to be a key factor in electrotonic modulation of AP recovery, ${ }^{3}$ our new three-dimensional AP representation shows for the first time what we defined as ARRP. The fact that, during this phase, repolarization is auto-regenerative, implies that the membrane at this time acts as an active source of repolarizing current. This has never been shown before in vivo nor in silico. The finding needs to be tested and confirmed at the tissue level, where electrotonic current flowing between cells should be taken into account as well. The existence of ARRP strongly suggests that cardiac ventricular repolarization is not, as usually described, a mere recovery of resting conditions after the active depolarization, but rather an active process. The source of autoregenerativity is very likely provided by the negative slope of the currentvoltage relationship of the inwardly rectifying potassium current $\mathrm{I}_{\mathrm{K} 1}$, which plays a major role in the late phase of cardiac AP repolarization. $\mathrm{I}_{\mathrm{K} 1}$ is expressed in many other excitable cell types, and this suggests a role for ARRP in modulating repolarization also in biological substrates different from the cardiac one, which is examined here.

\section{References}

1. Zaniboni M, Riva I, Cacciani F, Groppi M. How different two almost identical action potentials can be: a model study on cardiac repolarization. Math Biosci 2010;228:56-70.

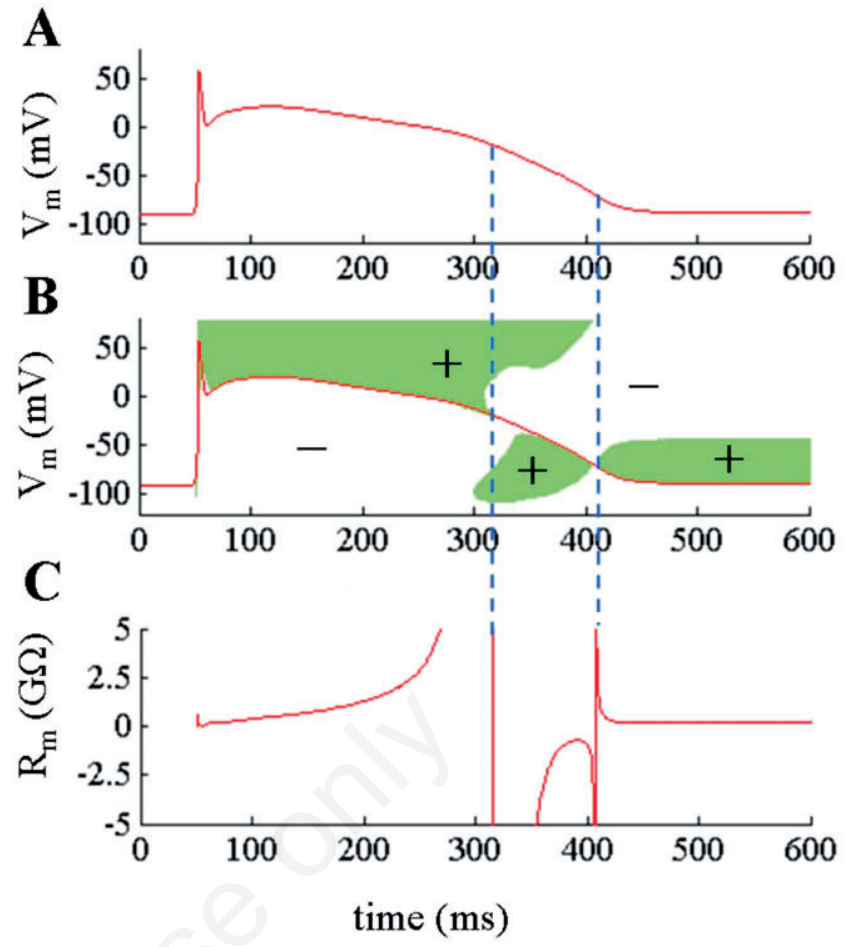

Figure 3. The three-dimensional surface derived for the Priebe $\&$ Beuckelmann action potential model (A) is represented here as a contour plot, where only negative (white) and positive (green) sign of $I_{m}$ is shown (B). The inverse of the slope of the $I_{m}$ surface along $I_{m}-V_{m}$ slices provides the time course of $R_{m}(C)$ during the action potential. The region where $R_{m}$ is negative is marked by the two blue dotted lines across the three panels.

2. Cherry EM, Fenton FH. A tale of two dogs: analyzing two models of canine ventricular electrophysiology. Am J Physiol-Heart C 2007;292:H43-55.

3. Zaniboni M, Pollard AE, Yang L, Spitzer KW. Beat-to-beat repolarization variability in ventricular myocytes and its suppression by electrical coupling. Am J Physiol-Heart C 2000;278:H677-87.

4. Zaniboni M. 3D current-voltage-time surfaces unveil critical repolarization differences underlying similar cardiac action potentials: A model study. Math Biosci 2011;233:98-110.

5. Zaniboni M. Late phase of repolarization is autoregenerative and scales linearly with action potential duration in mammals ventricular myocytes: a model study. IEEE T Bio-Med Eng 2012;59:226-33.

6. Zaniboni M. Heterogeneity of intrinsic repolarization properties within the human heart: new insights from simulated threedimensional current surfaces. IEEE T Bio-Med Eng 2012;59:237280.

7. Priebe L, Beuckelmann DJ. Simulation study of cellular electric properties in heart failure. Circ Res 1998;82:206-23. 